\title{
Pratiques plurielles de la science : Pierre Galland de Cherveux, géomètre au début du XIXe siècle
}

\author{
Jérôme Lamy
}

\section{(C) OpenEdition}

Édition électronique

URL : http://journals.openedition.org/abpo/2328

DOI : $10.4000 / a b p o .2328$

ISBN : 978-2-7535-1852-0

ISSN : $2108-6443$

Éditeur

Presses universitaires de Rennes

Édition imprimée

Date de publication : 30 mars 2012

Pagination : 145-156

ISBN : 978-2-7535-1850-6

ISSN : 0399-0826

\section{Référence électronique}

Jérôme Lamy, «Pratiques plurielles de la science : Pierre Galland de Cherveux, géomètre au début du XIXe siècle », Annales de Bretagne et des Pays de l'Ouest [En ligne], 119-1 | 2012, mis en ligne le 30 mars 2014, consulté le 01 mai 2019. URL : http://journals.openedition.org/abpo/2328 ; DOI : 10.4000/ abpo.2328 


\title{
Pratiques plurielles de la science : Pierre Galland de Cherveux, géomètre au début du XIX ${ }^{\mathrm{e}}$ siècle ${ }^{1}$
}

\author{
Jérôme LAMY \\ Post-doctorant au Laboratoire Interdisciplinaire, Solidarités, Sociétés, Territoire \\ (UMR 5193), Centre d'Anthropologie Sociale - Université Toulouse II Le Mirail
}

Pierre Galland est né à Cherveux, commune des Deux-Sèvres au nord de Niort, le 22 novembre $1757^{2}$, et il y est mort le 14 décembre $1837^{3}$. Les états de la population dressés dans le village en 1816 et 1822 indiquent qu'il exerce la profession de géomètre ${ }^{4}$. Son nom est inconnu ou presque des historiens des sciences. Il n'a pas été associé à une découverte majeure qui aurait marqué une discipline scientifique. Bien qu'il se soit essayé à la construction de machines innovantes, ses productions techniques ont été oubliées ou ignorées. Pierre Galland est donc un parfait inconnu qui a tenté à plusieurs reprises de s'inscrire dans une démarche scientifique en soumettant ses travaux à ses pairs, en s'impliquant dans une dynamique d'accumulation des connaissances et en proposant des inventions scientifiques et techniques. Il a ainsi développé des raisonnements sur la chimie, construit des machines optiques et proposé des écoles de géométrie locale pour les opérations de cadastre.

L'objectif de cet article n'est pas de retracer la totalité du parcours savant de Pierre Galland, ni de réhabiliter un savant méconnu. Nous proposons plutôt de considérer ce géomètre comme un problème historiographique. Comment pouvons-nous rendre compte, dans une démarche socio-historique, de pratiques scientifiques variées, parfois rejetées, parfois

1. Une première version de ce texte a été présentée en 2009 lors du $134^{\mathrm{e}}$ Congrès national des sociétés historiques et scientifiques qui s'est tenu à Bordeaux. Je remercie Sébastien Jahan, Pascale Quincy, Arnaud Saint-Martin, Volny Fages et Gérard Emptoz pour leurs commentaires, Claude Guicheteau pour ses indications archivistiques, ainsi que Jean-Pierre Petiot pour la traduction du texte de Goethe.

2. Arch. mun. de Cherveux, registre d'état civil, 1743-1762.

3. Arch. mun. de Cherveux, registre des décès, 1822-1842.

4. Arch. mun. de Cherveux, état de la population de la commune de Cherveux au $1^{\mathrm{er}}$ janvier 1822. État de la population de la commune de Cherveux, $1^{\mathrm{er}}$ janvier 1816. 
acceptées? Quel cadre d'intelligibilité permet de restituer les différentes pratiques savantes d'un géomètre de province du début du XIX ${ }^{\mathrm{e}}$ siècle dans leur complexité et leurs contradictions? Comment expliquer la diversité des arènes de réception et les formes variées de légitimation des savoirs? Ce qui nous intéresse ici, ce sont les logiques historiques d'organisation, de structuration et de cohésion de la communauté scientifique au début du $\mathrm{XIX}^{\mathrm{e}}$ siècle. Pierre Galland prétend faire de la science, rédige des mémoires, sollicite les instances académiques, et s'inscrit dans une démarche pédagogique; nous proposons donc de cerner, à partir de ces démarches construites, les pratiques sociales et épistémiques qui peuvent se subsumer, à l'aube du XIX ${ }^{\mathrm{e}}$ siècle, sous le terme science.

La sociologie transversaliste développée par Terry Shinn fournit un cadre théorique pour envisager les modes organisationnels dans lesquels les savoirs sont produits. Il s'agit, dans une perspective praxéologique, de distinguer différents régimes de sciences et techniques ${ }^{5}$. Ces formes différentes de l'activité scientifique ne se cristallisent pas en idéaux-types figés et fonctionnalistes. L'approche transversaliste propose de cerner des régimes de pratiques qui, à un moment donné, fixent un état précis de l'organisation des différentes manières de connaître. L'ensemble ne relève pas du système achevé, ni de la synthèse totalisante; bien au contraire, les régimes se transforment et les interactions qu'ils peuvent avoir se modifient également.

Il est possible de lire les travaux, les productions et les démarches de Pierre Galland à la lumière de la sociologie transversaliste en dégageant les régimes de sciences dans lesquels ils s'engagent. Au début du XIX ${ }^{\mathrm{e}}$ siècle, les pratiques scientifiques dans lesquelles s'implique le géomètre des DeuxSèvres s'organisent autour de trois régimes ${ }^{6}$ : le régime disciplinaire (centré sur un référentiel pratique et théorique strictement délimité par une communauté bien définie), le régime utilitaire (visant à la diffusion plus ou

5. SHINN, Terry, "The Bellevue grand électroaimant, 1900-1940. Birth of a researchtechnology community ", Historical Studies in the Physical Sciences, vol. 24, 1993, p. 157187. SHINN, Terry, "Change or mutation? Reflections on the foundations of contemporary science ", Social Science Information, vol. 38, n 1, 1999, p. 149-176. SHINN, Terry, "Axes thématiques et marches de diffusion ", Sociologie et societies, vol. 32, n 1, 2000, pp. 43-69. SHINN, Terry, "Formes de division du travail scientifique et convergence intellectuelle ", Revue Française de Sociologie, t. 41, n 3, p. 447-473. SHInN, Terry, JoERGEs, Bernward, "The transverse science and technology and technology culture. Dynamics and roles of research-technology ", Social Science Information, vol. 41, n 2, 2002, p. 207-251. SHINN, Terry et Ragouet, Pascal, Controverses sur la science. Pour une sociologie transversaliste de l'activité scientifique, Paris, Raisons d'agir, 2005.

6. L'approche transversaliste comprend deux autres régimes que nous n'évoquons pas ici car ils ne concernent pas Pierre Galland : le régime technico-instrumental renvoie à la conception et à la construction d'instruments génériques (comme par exemple l'utracentrifugeuse de Jesse Beams) circulant entre les régimes, le régime transitaire permet de modéliser la carrière des chercheurs passant d'une discipline à une autre pour y chercher des instruments ou des concepts (ShInN, Terry et RAGOUET, Pascal, Controverses sur la science..., op. cit., p. 168-178). 
moins large d'une innovation scientifique) et le régime officiel (assurant le bien commun). Nous détaillerons d'abord les conceptions scientifiques de Galland, puis nous examinerons comment ses propositions théoriques et pratiques s'intègrent ou non dans les régimes de sciences qu'il vise. Enfin, nous analyserons ses propositions relatives aux écoles de géométrie pour l'activité cadastrale.

\section{Des conceptions scientifiques datées?}

En 1804, Pierre Galland adresse à l'Athénée, société savante de Niort, un mémoire intitulé Essai d'un système d'héliophlogie ou Mémoire sur l'Application combinée des lois de la Diophlogie et de la Cataphlogie à l'Économie des combustibles. Dans l'introduction, le géomètre précise qu'il a dû inventer de nouveaux termes (héliophlogie, diophlogie, cataphlogie) car " la matière qu['il] ébauche [lui] paroît neuve " et " les expressions [lui] ont manqué ${ }^{7}$ ". Le mémoire s'ouvre par une longue présentation des connaissances antiques sur la lumière. Galland soutient que l'" Antiquité savante n'ignorait point les lois de la Lumière simple, ni de la lumière ignée passant obliquement d'un milieu dans un autre plus ou moins dense ${ }^{8}$ ». Il ne fait aucun doute pour lui que « les Égyptiens fesoient usage de Télescopes, puisqu'ils découvroient d'une haute tour d'Alexandrie les vaisseaux qui en étoient éloignés de 600 milles ". Par la suite, la cupidité, l'ignorance et la tyrannie ont fait disparaître " les vrais sages, les vrais savants, les vertus et les sciences elles-mêmes du dessus de la Terre ". Lorsque Giambattista della Porta, $\mathrm{au} \mathrm{XvI}^{\mathrm{e}}$ siècle, expose en termes obscurs ce qui semble être les principes de la lunette, il ne fait que les " recréer ${ }^{9}$ ". Cette longue digression historique permet également à Galland de fustiger Newton dont les " erreurs ont peut-être plus retardé le progrès des Sciences que ses grandes découvertes ne les ont avancés ${ }^{10} "$. Cette assertion se double d'une anglophobie affirmée en pleine troisième guerre de la coalition : les Britanniques sont accusés par Galland d'être des "Forbans [...] accapareurs de tout " qui " accapareront encore la gloire et le profit de toutes [les] Modernes découvertes ${ }^{11}$ ".

En redéployant une chronologie historique qui fait des Anciens les précurseurs oubliés des connaissances optiques, Galland signale la singularité de son mémoire qui ne s'inscrit pas dans le cadre d'une optique newto-

7. Arch. dép. des Deux-Sèvres, $\mathrm{F}^{\mathrm{n}-\mathrm{a}}$ 1513, Pierre Galland, Essai d'un Systême d'héliophlogie, an XIII.

8. Ibidem.

9. Ibid.

10. Ibid.

11. Ibid. Dans une lettre adressée au Journal des Deux-Sèvres et publiée en feuilleton, Pierre Galland s'en prend une nouvelle fois aux savants anglais : il revendique, pour la France, l'invention des verres périscopiques (Journal des Deux-Sèvres, politique, littéraire, commercial de la société d'agriculture et de l'athénée, samedi 11 mai 1816, n 19, p. 151. Ibid, samedi 25 mai $1816, \mathrm{n}^{\circ} 21$, p. 169.Ibid, samedi $1^{\mathrm{er}}$ juin $1816, \mathrm{n}^{\circ} 22$, p. 179. Ibid, samedi 29 juin 1816, $\mathrm{n}^{\circ} 26$, p. 209. Ibid, samedi 6 juillet $1816, \mathrm{n}^{\circ} 27$, p. 217). 
nienne mais pose, au contraire, les bases d'une nouvelle science. Toutefois, il ne s'agit pas d'opter pour une rupture complète avec les pratiques et les connaissances du siècle précédent, mais bien davantage de présenter un ensemble de savoirs inédits, qui se placent à côté, en parallèle, de ceux déjà produits et reconnus. Le géomètre reconnaît ainsi qu'il appelle " comme tout le monde Sciences Optiques [...] toutes les connoissances acquises qui se rapportent à la Lumière et à la vision ${ }^{12}$ ". Dans le même temps, il désigne sous le terme "sciences héliophlogiques celles qui se rapportent uniquement aux propriétés et aux effets d'ignition des verres et des miroirs ardens Exposés au Soleil ${ }^{13}$ ". En ce sens les deux sciences optiques et héliophlogiques ont recours aux mêmes outils (verres et miroirs) mais elles n'utilisent pas les mêmes lois. La suite du mémoire pose un problème qui met en lumière l'aberration produite par la sphéricité des verres. Galland interrompt son essai avant la deuxième partie; il explique que " plusieurs occupations et quelques voyages indispensables ont souvent interrompu et beaucoup retardé la rédaction de cette première partie ". Il se dit contraint de reporter la mise au point de la seconde partie à plus tard et promet de détailler le fonctionnement de machines héliophloges " capables de chauffer les appartemens, les Bureaux, les cabinets de travail, en hiver aux moindres rayons de Soleil ${ }^{14}$ ".

Nous ne disposons pas de la deuxième version de ce texte, qui comprenait les détails pratiques des propositions de Pierre Galland ${ }^{15}$. En revanche, le mémoire visant à obtenir du ministère du Commerce un brevet et la version imprimée de ce brevet nous sont parvenus et permettent de mieux cerner la circulation des productions scientifiques de Galland. La première version de l'Essai d'un système d'héliophlogie a été envoyée, par l'Athénée de Niort, à la "Classe des sciences Physiques et Mathématiques [... $]^{16}$ " de l'Institut. Le géomètre a également transmis ses travaux au journal Le Citoyen français du 6 floréal an XII ${ }^{17}$. La demande de brevet est co-signée avec Marie-Nicolas Chamblant présenté comme " bombeur de verre " et "Ingénieur Opticien ". Le texte reprend et développe, sur plus de 80 feuillets, les grandes lignes du mémoire soumis à l'Athé-

12. Arch. dép. des Deux-Sèvres, $\mathrm{F}^{\mathrm{n}-\mathrm{a}}$ 1513, Pierre GALLAND, Essai..., op. cit., an XIII.

13. Ibidem.

14. Ibid.

15. Galland assure que les rapports concernant son mémoire ont été reproduits dans " les feuilles du Journal et des Séances de l'Athénée " dont nous n'avons pu trouver la trace aux Archives départementales de Deux-Sèvres (Arch. de l'Institut National de la Propriété Industrielle, 1BA 757, Dossier Galland et Chamblant). Toutefois, le Journal du département des Deux-Sèvres reproduit, en 1804, une présentation du dispositif de Galland qui indique clairement que le manuscrit a bien été envoyé à l'Athénée de Niort (Journal du département des Deux-Sèvres, n ${ }^{\circ} 53,30$ prairial an XII [19 juin 1804]), p. 426-428 (Je remercie Claude Guicheteau pour cette indication).

16. Arch. de l'Institut National de la Propriété Industrielle, 1BA 757. GALLAND, Pierre et Chamblant, Marie-Nicolas Analyse des Mémoires d'un nouveau Système d'Héliophlogie et d'optique, s.d.

17. Ibidem. 
née de Niort. Surtout, Galland précise les soubassements théoriques de la science héliophlogique qu'il propose de fonder. La lumière héliophlogique est distincte de la " pure lumière solaire [...], simplement éclairante " et traversant " l'espace immense et extrêmement rare [...] sans résistance et sans réfraction depuis le soleil jusqu'à la surface extérieure de l'atmosphère ${ }^{18}$ ". La lumière héliophlogique, elle, est " tout à la fois éclairante, colorante et calorifique. Elle n'existe que dans l'atmosphère; elle y est une combinaison de la lumière solaire et des principes du feu désignés par Stahl sous le nom de Phlogistique, dont l'air et la terre sont les seuls réservoirs". C'est la densité de l'atmosphère qui donne à la lumière héliophlogique son " caractère remarquable " et qui explique les différences entre les lois de l'héliophlogie et de la science optique ${ }^{19}$. Galland s'appuie sur la théorie du phlogistique développée par le chimiste allemand Georg Ernst Stahl (16591734). Nous ignorons quelles sont ses sources précises; Lavoisier n'est pas mentionné, ce qui laisse ouverte l'interprétation du positionnement théorique de Galland.

Les travaux de Pierre Galland sur l'héliophlogie et son souci de fonder un nouveau corps de savoirs, à côté de ceux formant l'optique, illustrent la puissance et la domination du régime disciplinaire dans l'ordre des pratiques. Le mémoire soumis à l'Athénée de Niort puis à l'Institut ainsi que la demande de brevet révèlent une prétention théorique marquée. Nous ignorons les critiques exactes portées par l'Institut à l'endroit de sa conception héliophlogique de la lumière. Il n'en demeure pas moins que les références au phlogistique de Stahl n'ont plus cours dans la communauté savante vingt ans après les travaux de Lavoisier.

\section{Contraintes du régime disciplinaire et marchés de diffusion du régime utilitaire}

Le travail théorique de Galland est articulé à une invention qu'il tente, avec l'aide de l'opticien Chamblant, de faire connaître. Deux régimes de sciences sont ainsi mobilisés par le géomètre : régis par des normes différentes, ils renvoient à des procédures de validation distinctes.

Le premier de ces régimes, le plus ancien et le plus établi aussi, est le régime disciplinaire. Les disciplines sont structurées autour d'institutions assez facilement identifiées (académies, laboratoires, observatoires, publications) et s'inscrivant dans la durée. Les disciplines produisent d'importantes traces écrites et matérielles qui facilitent leur analyse. Les productions du régime disciplinaire concernent principalement des écrits (articles ou ouvrages), diffusés dans des espaces éditoriaux particuliers et soumis à l'approbation des pairs ${ }^{20}$. Le deuxième régime que l'on peut

18. Ibid.

19. Ibid.

20. Shinn, Terry et Ragouet, Pascal, Controverses sur la science..., op. cit., Paris, Raisons d'agir, 2005, p. 165-166. 
repérer dans l'activité de Pierre Galland est le régime utilitaire. Il est, au début du XIX ${ }^{\mathrm{e}}$ siècle, composé d'une population hétérogène d'ingénieurs, d'experts et de fabricants d'instruments. Ces praticiens de la science articulent savoirs théoriques et applications pratiques. Le marché de diffusion du régime utilitaire concerne à la fois des écrits spécialisés, mais aussi la production des normes et d'inventions.

Les développements conceptuels de Galland forment les prolégomènes à un ensemble de recherches pratiques qui, toutes, mettent en évidence l'importance et l'inconvénient des effets des aberrations de sphéricité des verres lenticulaires : "Les pouvoirs grossissant et diminuant des verres de l'ancien système ne sont pas les mêmes sur tous les points de leurs surfaces $^{21}$. " Le mémoire livré au ministère du Commerce est saturé d'informations et de projets associés. Ainsi, le géomètre imagine un " cabinet de travail qui serait tout à la fois Catadioplogique et catadioptrique " et pourrait témoigner dans son architecture de " la gloire et [de] la munificence $^{22}$ " de l'Empereur. Le nouveau système proposé par Galland et l'opticien Chamblant, qui fait plus directement l'objet de la demande de brevet, repose sur la fabrication et l'usage de " carreaux dioptriques". Il s'agit, pour chaque verre, de "deux segmens carrés du même cylindre, disposés parfaitement d'équerre, et réunis en opposition d'axes et de surfaces ${ }^{23}$ ". Ces carreaux auraient la propriété de se comporter comme des verres convexes et concaves ordinaires " mais avec cette différence [...] qu'ils transmettent et peignent les objets d'une manière uniforme par tous les points de leurs surfaces. Ils ne produisent aucune aberration déformative ${ }^{24}$ ". Les applications envisagées sont nombreuses pour corriger la presbytie, pour améliorer les lunettes d'opéra, les loupes, les microscopes, les télescopes astronomiques. Combiner plusieurs de ces carreaux à des miroirs ardents permet de produire une source de chaleur " nécessaire aux usages de la vie, des sciences et des $\operatorname{arts}^{25}$ ".

L'avis du Comité consultatif des manufactures et du commerce, rattaché au ministère de l'Intérieur, en date du 12 novembre 1812, estime que la présentation des procédés est " noyée dans beaucoup de choses étrangères au sujet \& la plupart erronées ". Le brevet d'invention ne pourra être accordé que si les deux impétrants font parvenir " une description plus simple de leurs procédés de fabrication et qui, en même temps [soit] débarrassée de toute Théorie étrangère ${ }^{26}$ ". Nous ne disposons pas de la version remaniée

21. Arch. dép. des Deux-Sèvres, $\mathrm{F}^{\mathrm{n}-\mathrm{a}} 1513$, Brevet d'invention, notice du système d'optique et d'héliophlogie, s.d.

22. Arch. de l'Institut National de la Propriété Industrielle, 1BA 757, GALLAND, Pierre et Chamblant, Marie-Nicolas, Analyse des Mémoire..., op. cit., s.d.

23. Arch. dép. des Deux-Sèvres, $\mathrm{F}^{\mathrm{n}-\mathrm{a}}$ 1513, Pierre Galland, Marie-Nicolas Chamblant, Brevet d'invention, Notice du systême d'optique et d'héliophlogie, s.d.

24. Ibidem.

25. Ibid.

26. Arch. de l'Institut National de la Propriété Industrielle, 1BA 757, ministère des Manufactures et du Commerce, Comité consultatif, séance du 12 novembre 1812. 
de la demande de brevet. Les modifications ont probablement satisfait le Comité consultatif puisque le 15 juin 1813, Galland et Chamblant obtiennent un " brevet d'invention de quinze ans " pour le " système d'héliophlogie et d'optique ${ }^{27} "$.

La circulation de l'invention se mesure d'abord aux mentions dans les publications techniques ${ }^{28}$. Le secrétaire du Conservatoire Royal des Arts et Métiers signale le nouveau procédé dans son Guide des Artistes, rédigé en $1818^{29}$. Les Archives des découvertes et inventions nouvelles évoquent également les " carreaux dioptriques ${ }^{30}$ " de Pierre Galland. Enfin, le brevet est intégralement imprimé dans la Description des machines et procédés de $1828^{31}$. L'invention du géomètre et de l'opticien n'est pas confinée au cadre national. Georg Altmütter professeur de technologie à l'Institut Polytechnique de Vienne s'y réfère en 1818, de même que David Brewster à Édimbourg. Airy, le célèbre astronome de Greenwich, y fait une vague allusion $^{32}$.

Les carreaux dioptriques sont également discutés par la première classe de l'Institut à laquelle Galland avait fait parvenir son mémoire initial. Nous ne disposons pas du rapport des commissaires nommés et leur expertise n'apparaît pas dans les procès-verbaux de l'Institut. Toutefois, l'article "Besicle " de l'Encyclopédie méthodique, daté de 1816, rend compte du processus académique sanctionnant l'invention du géomètre et de l'opticien. L'auteur de la notice décrit brièvement le procédé et précise, non sans ironie, que l'annonce d'une résolution du problème de l'aberration de sphéricité avait agrégé " des partisans " à Galland et Chamblant et que "des journaux parlèrent [du système] avec éloge ". Mais, c'est lorsque les deux hommes mandèrent des commissaires de la première classe de l'Institut, que " se termina leur succès ". Le carreau dioptrique examiné " avec soin ", n'avait en fait " aucun avantage sur celui des segmens de sphère tandis qu'il présentoit beaucoup de diffi-

27. Bulletin des lois du Royaume de France, $8^{\mathrm{e}}$ série,t. II, Paris, Imprimerie Royale, 1825, p. 344 .

28. L'invention de Galland et de Chamblant a connu une certaine diffusion puisqu'elle est citée par Goethe dans son journal de 1818. L'auteur allemand cite, en décembre " Die Gläser nach der neuen Methode von Galland und Cherveux " (" les verres selon la nouvelle méthode de Galland et Cherveux ", confondant le lieu de résidence de Galland et un co-inventeur). GOETHE, Johann, Goethes Tagebücher, vol. 6, Weimar, H. Bohlau, 1894, p. 272.

29. ARMONVILE, J.-R., Le Guide des Artistes ou répertoire des arts et manufactures, Paris, Imprimerie de Chaigniau aîné, 1818, p. 266.

30. Archives des découvertes et des inventions nouvelles faites dans les Sciences, les Arts et les Manufactures tant en France que dans les Pays étrangers pendant l'année 1813, Paris, chez Treuttel et Würtz, 1814, p. 341.

31. Description des machines et procédés cosignés dans les brevets d'invention, de perfectionnement et d'importation, t. XVI, Paris, 1828, p. 324-347.

32. Sur la circulation européenne des travaux de Galland et de Chamblant, voir : VoN RoHR, Moritz, "Contributions to the history of english opticians in the first half of the nineteenth century with special reference to spectacle history ", Transaction og the optical society, vol. XXVIII, $\mathrm{n}^{\circ}$ 3, 1926-1927, p. 133. 
cultés dans sa construction ". L'auteur de l'article reconnaît "que les verres cylindrés n'avoient pas d'aberration de sphéricité ", ce qui était précisément l'objectif de Galland et de Chamblant. Toutefois, les carreaux dioptriques ont une autre " aberration aussi désavantageuse, celle de cylindricité, qui déformoit également les objets ${ }^{33}$ ".

La réception des travaux de Galland dans le cadre du régime disciplinaire est rendue compliquée par les différents niveaux de lecture qu'ils imposent. Ainsi, les réflexions et les réalisations de Galland et Chamblant sur la suppression de sphéricité peuvent avoir convaincu les membres de l'Institut, mais la découverte, sur leurs verres, d'une aberration de cylindricité démonétise leurs propositions. En somme, le géomètre et l'ingénieur restent au seuil de la reconnaissance scientifique. Leurs recherches sont examinées mais non point légitimées dans le processus de sanction académique. La dimension européenne de l'évaluation confirme le rejet des carreaux dioptriques.

Les expérimentations menant à la construction des carreaux dioptriques, le travail du verre effectué avec l'ingénieur Chamblant, la rédaction d'une demande de brevet et les relais à la Société d'Encouragement pour l'Industrie Nationale ${ }^{34}$ témoignent d'une démarche scientifique visant à la production et à la commercialisation d'un procédé technique qui dépasse le seul cadre de la discipline optique et s'inscrive clairement dans le régime utilitaire. Galland précise dans le brevet qu'il dépose les multiples applications des carreaux dioptriques, des microscopes aux lunettes d'opéra. Le comité des manufactures et du commerce qui évalue la première version du brevet des verres catadioptriques souligne que les auteurs ont noyé leur démonstration dans des considérations théoriques hors de propos. Dans le même temps, les rédacteurs de L'Encyclopédie méthodique ironisaient sur le succès médiatique de Galland et Chamblant. Les deux hommes semblent ne maîtriser qu'imparfaitement leurs discours devant les audiences auxquelles ils s'adressent. Les régimes de sciences diffusent des productions spécifiques (articles, brevet selon les cas) qui visent un public précis (membres de l'Institut, ingénieurs). En développant un long argumentaire dans une demande de brevet ou en réclamant une expertise académique après avoir obtenu des soutiens dans la presse, Galland et Chamblant opèrent une rupture de cadre ${ }^{35}$, c'est à dire que les productions qu'ils soumettent ne sont pas adaptées aux audiences visées. La rédaction d'un brevet est une pratique socialement cadrée qui suppose la maîtrise de codes lexicaux et rhétoriques relativement stabilisés au début du XIX ${ }^{\mathrm{e}}$ siècle : le déploiement

33. Encyclopédie Méthodique, Physique, T. II, Paris, Agasse, 1816, p. 103 (pour toutes les citations concernant l'article "Besicle").

34. Les auteurs indiquent que leur invention a été examinée par la Société d'encouragement pour l'industrie nationale. Il semble que Galland et Chamblant aient effectivement envoyé leur proposition à la Société et que celle-ci l'ait faite parvenir au Comité des Arts Mécaniques (Bulletin de la Société d'Encouragement pour l'Industrie Nationale, 12 année, $\mathrm{n}^{\circ}$ CVIII, juin 1813, p. 127. Je remercie Claude Guicheteau pour cette indication).

35. Goffman, Erwin, Les cadres de l'expérience, Paris, Éditions de Minuit, 1974, p. 340. 
d'une conception théorique nouvelle de la lumière n'entre pas, au sein du régime utilitaire, dans les pratiques attendues. De même, la demande d'une sanction académique après la rédaction d'un article dans Le Citoyen français témoigne d'une certaine méconnaissance des usages dans la présentation des résultats scientifiques.

\section{Les balbutiements du régime officiel}

Le troisième régime dans lequel s'engage Galland est, le régime officiel ${ }^{36}$. Il concerne l'ensemble des pratiques scientifiques et bureaucratiques visant à la satisfaction du bien commun. Fortement cadré par la loi et décliné sous la forme d'un service public de la science, le régime officiel s'élabore peu à peu depuis la fin du XVII ${ }^{\mathrm{e}}$ siècle.

L'activité scientifique de Pierre Galland ne se limite pas aux inventions optiques. Nous n'avons cependant que très peu d'informations sur son activité quotidienne de géomètre. L'opération cadastrale napoléonienne permet d'envisager sa conception du travail d'arpentage et la manière dont il s'inscrit dans un projet impérial de maîtrise et de contrôle des territoires. Pendant la Révolution, la Constituante avait œuvré pour la mise en place d'un cadastre articulé à la question des contributions foncières. Le projet est recentré sur les surfaces cultivées en 1802, puis la loi du 15 septembre 1807 " opte finalement pour un cadastre parcellaire ${ }^{37}$ " qui doit indiquer la dimension et le revenu de chaque propriété.

L'arpentage complet de chaque commune suppose la mobilisation de nombreux géomètres à travers le pays. Pierre Galland est sollicité par le préfet des Deux-Sèvres pour effectuer des opérations de cadastre dans son canton. Les lettres qu'il fait parvenir à l'administration consulaire montrent ses doutes sur la réalisation de l'entreprise et son souci d'être justement rémunéré pour une tâche importante. Galland accepte d'abord de se charger de "l'Arpentement de masse de chaque commune " du canton; il ajoute toutefois que s'il s'agit de "mesurer en détail chaque propriété particulière [...] le travail deviendroit beaucoup plus long et plus dispendieux ${ }^{38}$ ". La rémunération constitue également un point d'achoppement pour s'engager dans le projet; il n'acceptera pas de commencer l'arpentage « sans que le

36. SAINT-MARTIN, Arnaud, L'office et le télescope. Une sociologie historique de l'astronomie française, 1900-1940, thèse de sociologie, Université Paris-Sorbonne, 2008, $\mathrm{f}^{\circ}$ 31-33. LAMY, Jérôme, SAINT-MARTIN, Arnaud, " La science au service de l'État. Éléments pour une étude du "régime officiel" de la science ", Séminaire Circulation transdisciplianire des savoirs et commensurabilité. La place des instruments conceptuels et matériels, EHESS, 20 février 2007.

37. OliveIRA, Mathieu de, "Un nouveau corps de fonctionnaires techniciens : les ingénieurs et géomètres du cadastre 1800-1830 ", dans : BourILLON, Florence, ClERGEOT, Pierre, VIVIER, Nadine (dir.), De l'estime au cadastre en Europe. Les systèmes cadastraux aux XIX et $X X^{e}$ siècles. Colloque des 20 et 21 janvier 2005, Paris, Comité pour l'histoire économique et financière de la France, 2008, p. 178.

38. Arch. dép. des Deux-Sèvres, 3P 3, lettre de Pierre Galland à la préfecture des DeuxSèvres, 20 floréal an IX. 
prix qui doit y être destiné [lui] soit assuré ». Galland réclame en outre le soutien d'un " ayde déjà instruit dans cette partie ${ }^{39}$ ". Les prix proposés lui semblent dérisoires au regard des difficultés d'une levée cadastrale fort complexe dans un territoire de bocage.

Le recrutement d'un personnel suffisamment qualifié pour effectuer les mesures et constituer les plans est une préoccupation du ministère des finances qui centralise les opérations. Un premier cours de géométrie destiné à former le personnel de l'opération cadastrale est ouvert le 27 nivôse an XII dans le département de la Seine ${ }^{40}$. L'enseignement des éléments essentiels de géométrie, d'arithmétique et de géodésie s'étend à vingt-trois départements en août 1804. L'ingénieur en charge d'organiser le cadastre et de centraliser les opérations d'arpentage dans le département des Deux-Sèvres suggère, dès le 30 messidor an X, que soit créée une école de géométrie qui pourrait donner " quelques élèves à ceux des géographes reconnus pour avoir les connaissances requises et en payant ces derniers en conséquence ${ }^{41}$ ». Peu de temps après, Pierre Galland propose au préfet de mettre sur pied une telle structure pédagogique. Il assure, le 5 floréal an XII, avoir senti la nécessité d'établir " des Écoles de géométrie-pratique sans lesquelles [...] il sera même impossible d'opérer le cadastre complet d'aucun département ${ }^{42}$ ". Il soumet un mémoire présentant les grandes lignes de l'école qu'il compte créer chez lui; il s'agira d'exposer toutes les opérations d'arpentage, de levée des plans et de cartographie, " conformément aux instructions du Bureau du Cadastre Général à Paris ". Un brevet pourrait couronner les impétrants; les plus doués pourraient être initiés à la stéréométrie et à la perspective ${ }^{43}$. Le projet est vivement soutenu par le préfet ${ }^{44}$, mais les archives manquent pour savoir si cette école a effectivement été créée.

L'opération cadastrale consulaire puis impériale s'inscrit dans une démarche scientifique contrôlée par l'État et définie par la loi, articulant questions fiscales, contrôle des territoires et souci du bien commun par la délimitation des propriétés ${ }^{45}$. Les géomètres qui opèrent les relevés des terrains et produisent les cadastres au début du XIX ${ }^{\mathrm{e}}$ siècle constituent les linéaments d'un service public de la science. Galland semble très

39. Arch. dép. des Deux-Sèvres, 3P 3, lettre de Pierre Galland à la préfecture des DeuxSèvres, 29 fructidor an IX.

40. OLIVEIRA, Mathieu de, "Un nouveau corps... ", op. cit., p. 179.

41. Arch. dép. des Deux-Sèvres, 3P 3. Observations de l'Ingénieur en chef sur le mode suivi dans l'arrondissement de la sous-préfecture de Melle pour l'arpentement des communes, 30 messidor an X.

42. Arch. dép. des Deux-Sèvres, 3P 3, lettre de Pierre Galland à la préfecture des DeuxSèvres, 5 floréal an XII.

43. Arch. dép. des Deux-Sèvres, 3P 3. Pierre GaLLAND, Cours de Géométrie pratique pour le cadastre du département des Deux-Sèvres, s.d.

44. Une note du préfet (datée du 27 vendémiaire an XIII) en marge du projet de Pierre Galland indique que le représentant de l'État " approuve les dispositions " de l'école et souhaite qu'elle soit relayée par le ministère (Ibidem).

45. Oliveira, Mathieu de, "Un nouveau corps... ", op. cit., p. 175-176. 
sensible à cette notion de service public. Il évoque, à plusieurs reprises dans ses lettres à la préfecture, son désir de devenir " arpenteur des forêts Nationales " afin de " continuer [s]es services pour la chose publique ${ }^{46}$ ". Son projet d'école de Géométrie pratique s'inscrit, dit-il, dans un vaste dessein gouvernemental ouvrant " un carrière immense aux Opérations Géométriques et une Perspective assurée aux Jeunes Gens qui voudroient y entrer $^{47}$ ". Que cette école ait ou non vu le jour importe moins que l'engagement de Galland dans un régime officiel de sciences qui délimite, à travers la routinisation des tâches d'arpentage et de déploiement du cadastre, les éléments articulés d'une pratique scientifique d'État centrée sur le bien commun $^{48}$.

La variété des pratiques scientifiques dans lesquelles Pierre Galland est engagé fournit un levier épistémologique puissant. En raisonnant dans le cadre de l'approche transversaliste, nous avons pu saisir les attentes du géomètre, son souci de défendre une théorie optique, l'irréductibilité des pratiques utilitaires et disciplinaires, les modes de diffusion et de réception des travaux académiques ainsi que la construction d'une science officielle du cadastre. Les droits d'entrée dans les différents régimes sont différents, les codes et les usages en vigueur supposent des apprentissages spécifiques et la réception des productions scientifiques dépend non seulement de la qualité intrinsèque des recherches, mais également de la capacité des prétendants à appréhender dans leur diversité, les normes et les règles de chaque régime. Ainsi, les pratiques scientifiques de Pierre Galland ne peuvent s'envisager sur le mode unique d'un savant de province méconnaissant les usages de la communauté savante. Elles peuvent être expliquées, dans une perspective transversaliste, comme des engagements plus ou moins maîtrisés, dans des registres de pratique différents.

46. Arch. dép. des Deux-Sèvres, 3P 3, lettre de Pierre Galland à la préfecture des DeuxSèvres, 20 floréal an IX.

47. Arch. dép. des Deux-Sèvres, Pierre Galland, Cours de Géométrie pratique pour le cadastre du département des Deux-Sèvres, s.d.

48. Nous devons ajouter qu'il existe un document aux Archives départementales des Deux-Sèvres intitulé Tableau d'une extrême simplicité, au moyen duquel, tout d'un coup et sans calcul, on convertit, en nouvelles, les anciennes Mesures agraires du département des Deux-Sèvres, pour une grandeur quelconque, depuis un Carreau ou centième de Boisselées inclusivement (Arch. dép. des Deux-Sèvres, Br 77/45). Ce tableau imprimé est signé Galland, mais il n'est pas absolument certain qu'il s'agisse bien de Pierre Galland. Dans l'hypothèse où c'est bien le géomètre de Cherveux qui a mis au point ce tableau, cela renforce son implication dans le régime officiel qui se déploie dans les pratiques métrologiques visant à fournir au plus grand nombre des repères cohérents dans l'emploi des mesures quotidiennes. 


\section{RÉSUMÉ}

Pierre Galland est géomètre dans un village près de Niort à la fin du $\mathrm{XVIII}^{\mathrm{e}}$ siècle et au début du $\mathrm{XIX}^{\mathrm{e}}$ siècle. Il n'a jamais publié dans une revue scientifique de l'époque, ni dialogué avec les autres savants de son époque. Ces seules activités scientifiques connues - outre son quotidien de géomètre sont un procédé optique (qu'il baptise héliopphlogie) déposé auprès de la Société d'Encouragement pour l'Industrie Nationale ainsi qu'une forte implication dans les opérations de cadastrage lancées sous le Consulat.

Le parcours de Pierre Galland, constitue un véritable problème historiographique intéressant. Cet article envisage donc, à travers l'approche transversaliste développée par Terry Shinn, de retracer les engagements de Pierre Galland dans les régimes disciplinaire, utilitaire et officiel pour faire science. Ce cadrage conceptuel permet une réflexion sur la multitude de productions qui échappent aux académies et aux surfaces éditoriales les plus connues, mais qui cherchent à s'inscrire dans les processus d'accumulation des connaissances.

\section{ABSTRACT}

Pierre Galland was a geometrician in a village near Niort, at the end of the $18^{\text {th }}$ century and the beginning of the $19^{\text {th }}$ century. He never published anything in any learned review of the time or debated with other scientists of his days. His only known scientific activities - outside his daily life as a surveyor-are an optical device (which he called heliopphlogie) registered with the Society for the Promotion of National Industry and a strong implication in the land-surveying operations carried on under the Consulate.

The life story of Pierre Gaillard is a really interesting historiographic problem. So, using Terry Shinn's transversalist approach, this article purposes to follow Pierre Gaillard's engagements in various domains, utilitarian and official, all leading to the progression of science. This conceptual framework allows us to reflect on the multitude of productions that are not to be found in the academies and the bestknown publications, but endeavour to contribute to the increase of knowledge. 